\title{
Performance analysis of multi-hop framed ALOHA systems with virtual antenna arrays
}

\author{
Chiara Buratti ${ }^{*}$, Alberto Zanella ${ }^{2}$ and Roberto Verdone ${ }^{1}$
}

\begin{abstract}
We consider a multi-hop virtual multiple-input-multiple-output system, which uses the framed ALOHA technique to select the radio resource at each hop. In this scenario, the source, destination and relaying nodes cooperate with neighboring devices to exploit spatial diversity by means of the concept of virtual antenna array. We investigate both the optimum number of slots per frame in the slotted structure and once the source-destination distance is fixed, the impact of the number of hops on the system performance. A comparison with deterministic, centralized re-use strategies is also presented. Outage probability, average throughput, and energy efficiency are the metrics used to evaluate the performance. Two approximated mathematical expressions are given for the outage probability, which represent lower bounds for the exact metric derived in the paper.
\end{abstract}

\section{Introduction}

Multiple-input multiple-output (MIMO) systems are well known for their capability to obtain high spectral efficiency in the presence of fading channels [1,2]. On the other hand, the need to install multiple antennas in portable devices can be problematic for economic and practical reasons. To extend the advantages of MIMO systems to devices characterized by a reduced number of antennas, the idea of deploying a virtual (or distributed) (V-MIMO) architecture appears to be very promising [3-5]. Cooperation among nodes is a fundamental aspect of V-MIMO systems, since nodes cooperate to create virtual antenna arrays (VAAs) [4]. V-MIMO systems take also advantage from the use of nodes equipped with two radio transceivers: a long-range wireless system, which can be used for inter-VAA communication, and a shortrange radio interface, which can be used for intra-VAA communication $[3,4,6]$.

Virtual MIMO systems can be applied in the context of both centralized and decentralized networks. The performance of virtual MIMO system applied to a cellularbased architecture in the presence of a limited amount of feedback information is evaluated in terms of throughputfairness trade-off [7]. The impact of channel estimation

*Correspondence: c.buratti@unibo.it

${ }^{1}$ RadioNetworks, DEl, University of Bologna, Bologna, Italy

Full list of author information is available at the end of the article errors on the performance of relay-assisted virtual MIMO systems is discussed in [8].

The case of multi-hop systems is of particular interest, as V-MIMO techniques can improve significantly the performance in terms of throughput compared to conventional single-input, single-output or SISO schemes [9-19]. In this contest, distributed radio resource assignment techniques, being much simpler, are to be preferred to centralized schemes. The topic of resource allocation, when deterministic (and centralized) multiple access control (MAC) techniques are used for inter-VAA communication, is studied in $[3,4,6,18]$. The overall resource pool, available for inter-VAA communication, is assumed to be fractionated into orthogonal radio resources for inter-VAA links in $[3,4,18]$, and the performance of the considered V-MIMO scheme is investigated. As far as distributed radio resource assignment techniques are considered, the impact of non-orthogonal reuse of radio resources in a multi-hop V-MIMO system with a fixed number of relays has been investigated in [6]. In that paper, the presence of interference caused by nonorthogonal reuse of resources has been quantified, and its effect on the source-destination performance has been studied.

It is well known that MIMO technology can improve the performance of conventional random-access schemes in terms of reliability and throughput. In particular, the use of MIMO detection techniques to combine signals sampled at successive time slots can reduce (or eliminate)

\section{Springer}

2013 Buratti et al: licensee Springer. This is an Open Access article distributed under the terms of the Creative Commons

Attribution License (http://creativecommons.org/licenses/by/2.0), which permits unrestricted use, distribution, and reproduction in any medium, provided the original work is properly cited. 
the effect of collisions. This important result has motivated the research of network diversity multiple-access (NDMA) schemes that can obtain a good performance when the channel coherent time is in the order of the packet slot duration [20,21]. Among the random access schemes, ALOHA has been recently proposed for MIMO systems in [22-24]. More precisely, a scenario composed of an access point (AP), and multiple transmitting nodes is considered in [22]. The key idea of [22] is that nodes use framed ALOHA (FA), but the possible multiple transmissions over the same slot are not treated as collisions. In the event of collision, some nodes are selected to act as relays and retransmit the signal they received during the collision in a specific time slot. The different signals received by the AP are then processed using standard detection strategies (i.e., using maximum likelihood techniques). To alleviate the performance degradation which occurs in NDMA schemes when coherent time is larger than the packet slot duration, an adaptive space-time diversity framed ALOHA scheme, which works with MIMO systems with two transmit and an arbitrary number of receive antennas, is proposed in [23]. Multiplexing/diversity MIMO trade-off is considered in the context of wireless local area networks performance optimization with MIMO channels [24]. The paper proposes a joint optimization of MAC layer parameters and the multiplexing-diversity trade-off at the physical layer. Two different MAC protocols are studied: carrier sense multiple access with collision avoidance (CSMA/CA) and slotted ALOHA. CSMA is also used in [25] for distributed scheduling in multi-hop networks with MIMO links. An adaptive CSMA protocol, which adjusts the link rate based on local queue information, is also proposed in [25].

To the authors' knowledge, there are no papers in the literature addressing the performance of multihop V-MIMO systems where random access-based MAC schemes are used for inter-VAA communication. Motivated by this consideration, a decentralized MAC scheme is considered in this paper. In particular, the resources are assigned to each hop independently, through a framed ALOHA procedure: the time axis is split into frames, each composed of a given number of slots. Assuming perfect synchronization among nodes, each VAA in the multi-hop chain selects one slot randomly, resulting in possible interference among VAAs selecting the same slot. It is well known that this approach provides worse performance than the case of a centralized procedure implementing a perfect deterministic reuse of the time slots. On the other hand, implementation of framed ALOHA is much simpler than that of a centralized algorithm since resources are independently selected by nodes at each hop. Centralized assignment of the resources, in fact, requires the exchange of many control packets among nodes, and it is not applicable in many cases, as for example wireless sensor networks $[26,27]$.

Network performance is evaluated in terms of ergodic capacity, outage probability, average throughput, and energy efficiency. The model presented in this paper is based on the works presented in $[28,29]$ and it represents a generalization and integration of those contained in the two conference papers.

The contribution of this paper can be summarized as follows:

- We showed the behavior of the outage probability by varying different system parameters, and compare framed ALOHA with a deterministic centralized assignment of radio resources.

- We identified the optimum number of slots per frame in the slotted structure of ALOHA;

- We evaluated the impact of the number of hops on the performance when the source-destination distance and the other system parameters are set.

- We discussed the trade-off between source-destination throughput and overall energy spent by nodes.

- We provided some upper bounds on the outage probability. Numerical results will show that these bounds are rather tight.

The remainder of the paper is organized as follows. Section 2 describes the channel model considered, the reference scenario, the framed ALOHA protocol, and the communication within the multi-hop VAA-based network. Section 3 provides the mathematical model to derive the ergodic capacity of the multi-hop V-MIMO system, while Section 4 reports the derivation of the performance metrics: outage probability, average throughput, and energy efficiency. Finally, Sections 5 and 6 report the numerical results and conclusions, respectively.

\section{System description and scenario}

\subsection{Notations}

Throughout the paper, vectors and matrices are indicated in bold, $\mathbf{I}$ is the identity matrix, $[\mathbf{A}]^{\mathrm{T}}$ denotes the transpose of $\mathbf{A} .\left\{a_{i, j}\right\}_{i, j=1, \ldots, M}$ is an $M \times M$ matrix with elements $a_{i, j}=\{\mathbf{A}\}_{i, j} . \mathbf{A} \oplus \mathbf{B} \triangleq \operatorname{diag}(\mathbf{A}, \mathbf{B})$ is the direct sum of matrices [30]. $\mathbb{E}\{\cdot\}$ denotes expectation, and $\mathbb{P}\{\mathcal{E}\}$ denotes the probability of the event $\mathcal{E} . \mathrm{u}(x)$ indicates the unit step function, equal to one for $x>0$ and zero otherwise.

\subsection{Propagation and connectivity models}

The channel model we consider accounts for the power loss due to propagation effects including a distancedependent path loss and random channel fluctuations. We assume that the received power, $P_{\mathrm{R}}$, is given by 


$$
P_{\mathrm{R}}=\frac{P_{\mathrm{T}} x^{-\beta} f}{k}
$$

where $P_{\mathrm{T}}$ is the transmitted power, $k$ is the average power loss at $x=1 \mathrm{~m}$, being $x$ the link distance, $\beta$ is the attenuation coefficient which commonly ranges from 2 to 5 ; finally, $f$ is a short-term random fading component. Rayleigh fading is considered for the inter-VAA links, so that $f$ is exponentially distributed with unit mean.

We define $L \triangleq k \cdot x^{\beta}$ as the averaged (with respect to fading) loss (in linear scale). By introducing the logarithmic scale, we obtain

$$
L[d B]=k_{0}+k_{1} \ln x,
$$

where $k_{0}=\zeta \ln k$ and $k_{1}=\zeta \cdot \beta$, where $\zeta \triangleq 10 / \ln 10$.

Note that, for each air interface (intra- and inter-VAA), we could have different values of the transmitted power $\left(P_{\mathrm{T}}\right)$ and propagation parameters $\left(k_{0}, k_{1}\right)$.

The channel is assumed to be ergodic. Under such assumption, the source-destination ergodic capacity can be considered as one of the main performance metrics [31].

\subsection{Reference scenario and assumptions}

We consider a multi-hop V-MIMO system (see Figure 1), where a source node (node $\mathrm{S}$ in the figure) transmits data to a destination node (node $D$ ), via multiple relay nodes $\left(R_{1}, R_{2}, . . R_{Z-1}\right)$, being $Z$ the number of hops in the network.

In the following, source, relays and destination nodes will be denoted as main nodes. The distance between the source main node and the destination main node will be denoted as $D_{\mathrm{SD}}$, while $d$ will be the distance between the main nodes of two subsequent VAAs in the multihop chain. We assume that adjacent VAAs are equally separated in distance (i.e., $d=D_{\mathrm{SD}} / Z$ ).

A random number of ancillary nodes is distributed in non-overlapped arbitrary areas, $\mathcal{A}$, around the main nodes, according to a homogeneous Poisson point process (PPP) [32]. According to such distribution, the probability of having one node in the infinitesimal area $\delta \mathcal{A}$ is $\rho / \delta \mathcal{A}$, where $\rho$ ' denotes the nodes' density [32]. Owing to the possible presence of mechanisms such as sleeping schedules implemented at nodes, only a subset of the ancillary nodes are simultaneously active; we denote $\rho$ as the density of the active nodes.

As largely adopted in the literature, we assume that nodes are equipped with two air interfaces: a shortrange air interface, which is characterized by a high data rate and low energy consumption and used for the communication within VAA (intra-VAA communication), and a long-range air interface, which is characterized by a lower data rate and used for the inter-VAA communication $[3,4,6]$.

The main and ancillary nodes may cooperate to create VAAs at the source (s-VAA), at the $Z-1$ relays $\left(r_{i}\right.$-VAA, with $i \in\{1, . ., Z-1\})$ and at the destination (d-VAA). In particular, we assume that an ancillary node could be part of the VAA only if the main node-ancillary node path loss, $L$, is lower than a given threshold, $L_{\mathrm{th}}{ }^{\mathrm{a}}$, representing the maximum loss tolerable by the short-range communication system. VAAs are formed before each data transmission. We assume that the path loss between main and ancillary node, $L$, is constant during each interVAA transmission phase. Short-range wireless interface is used to track possible changes in the propagation characteristics and identify the new set of collaborating nodes. The new set of cooperating nodes will be active during the subsequent transmission phase. Using the propagation model given in (1), the results that ancillary nodes may communicate with the main node only if their distance is lower than $d_{\mathrm{th}}=e^{\left(L_{\mathrm{th}}-k_{0}\right) / k_{1}}$ and the mean number of candidate cooperating nodes, denoted as $N$ hereafter, can be written as $\rho \pi d_{\mathrm{th}}^{2}$. We assume that the distances sourcerelay, relay-relay, and relay-destination are much larger than the distance between a main node and its cooperating nodes. With this assumption, the VAAs do not overlap and each ancillary node can communicate only with a unique main node. Moreover, depending on the shortrange air interface used, there could exists a maximum number of cooperating nodes a main node can actually
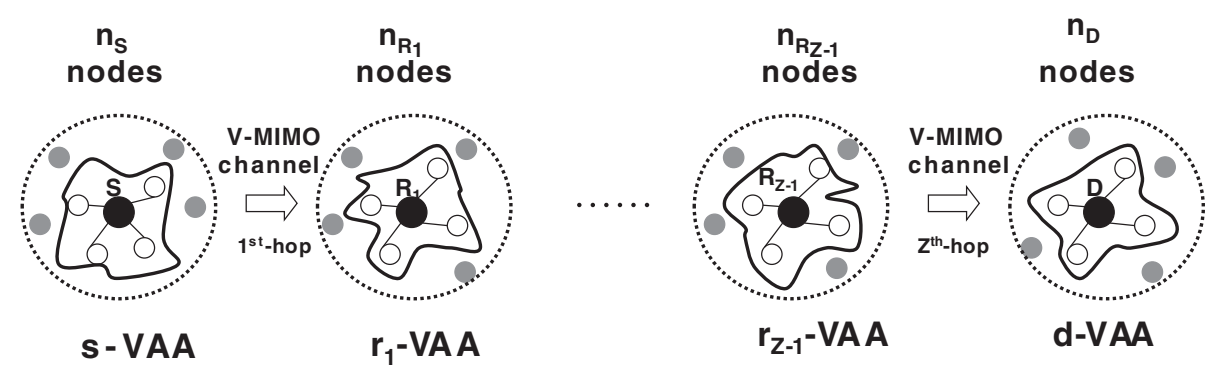

Figure 1 The virtual MIMO communication system. 
handle; this maximum number will be denoted as $M$ in the following. Finally, we assume that a decode and forward (DF) strategy is implemented at the relays [4].

\subsection{Framed ALOHA}

We assume that a framed ALOHA protocol is used by the different VAAs to access the channel [33]; the whole amount of radio resources available for inter-VAA communication is divided among $R$ unitary resources. In other words, each frame has $R$ time slots. Therefore, once a VAA has data to be transmitted, the main node of the VAA will select randomly one out of the $R$ available time slots in the next frame and will communicate the choice to its cooperating nodes. All nodes of a given VAA (cooperating nodes plus the main node) will simultaneously access the channel by using the same unitary resource, selected with probability $1 / R$. We also assume an out-of-band signalling mechanism is used for synchronization between the transmitter VAA and the receiver VAA.

Since time slot selection is made independently at VAA level, there is a nonzero probability that other links use the same time slot, causing interference.

\subsection{The communication in the multi-hop chain}

We assume that all the VAAs (s-VAA, $r_{i}$-VAAs (with $i \in$ $\{1, . ., Z-1\})$ and d-VAA) are formed before data transmission. During the phase of VAA creation, each main node selects the slot to the used for the inter-VAA communication. Large scale effects (i.e., path-loss) are supposed to be slowly varying compared to the data packet length. Under such assumption, the set of cooperating nodes remains constant during the packet transmission.

Using the short-range radio interface, the main nodes transmit a query to ancillary nodes, and those nodes that receive the query reply with an acknowledgement, becoming candidate cooperating nodes. Then, the main nodes randomly select $M$ nodes, among all the candidates, to cooperate with. Being ancillary nodes distributed according to a PPP, the number of candidate cooperating devices at a given node follows a Poisson distribution. The number of nodes actually cooperating at the source, the $i$ th relay and at the destination, will be denoted as $n_{\mathrm{S}}, n_{\mathrm{R}_{\mathrm{i}}}$, and $n_{\mathrm{D}}$, respectively.

Once all the VAAs are formed, the communication between source (main) node and destination (main) node is performed according to the following steps:

1. The source main node will transmit the data, together with the slot to be used, to the $n_{\mathrm{S}}$ cooperating nodes, using the short-range air interface (intra-VAA communication).

2. The $n_{\mathrm{S}}+1$ nodes of the s-VAA transmit data towards the first relay through the V-MIMO channel, using the long-range interface (inter-VAA communication).
3. The $n_{\mathrm{R} 1}+1$ nodes of the $r_{1}$-VAA cooperate to decode the received data, meaning that the data is send (through the short-range interface) from the $n_{\mathrm{R} 1}$ nodes to the main node, which will decode it.

4. The main node at the $r_{1}$-VAA sends the data to be forwarded, together with the slot to be used, to the $n_{\mathrm{R} 1}$ cooperating nodes, which will forward it towards the second relay.

5. The previous two steps are repeated until the data reach the last relay.

6. The $n_{\mathrm{D}}+1$ nodes of the $\mathrm{d}-\mathrm{VAA}$ receive data from the last relay and cooperate to decode it.

In this paper, we focus on inter-VAA performance, which uses long-range air interface. Data transmission between main and cooperating nodes can be performed either via broadcast transmission (all nodes receive all data), or using a given multiple access scheme; this aspect, which is related to intra-VAA communication, is out of the scope of this paper.

More specifically, the exchange of information between main node and ancillary node for signaling, synchronization and data transmission (intra-VAA transmission) uses the short-range radio interface. This solution also minimizes possible delays since the two transmission phases (intra- and inter-VAA communications) can occur in parallel. For this reason, delays or other possible overhead due to cooperation aspects have not been considered.

\section{Analysis of the ergodic capacity for a V-MIMO system}

\subsection{Capacity of a generic link}

In this section we focus on a generic hop (the $\ell$ th hop) of our scenario and assume that the link is affected by the presence of other $I^{(\ell)}$ VAAs, acting as interferers. We denote $n_{\mathrm{R}}^{(\ell)}$ as the number of nodes (cooperating and main) at the receiver VAA, $n_{\mathrm{T}_{\mathrm{j}}}^{(\ell)}$ with $j \in\left(0, \ldots, I^{(\ell)}\right)$ as the number of transmit nodes of the desired $(j=0)$ and of the interfering $\left(j=1, \ldots, I^{(\ell)}\right)$ VAAs. We denote $n_{\mathrm{T}_{I}}^{(\ell)}=\sum_{j=1}^{I^{(\ell)}} n_{\mathrm{T}_{j}}^{(\ell)}$ as the total number of transmit nodes interfering on the $\ell$ th hop. We assume that nodes belonging to the same VAA transmit the same amount of power. Under such hypothesis, the ergodic capacity in the $\ell$ th hop in the case of uncorrelated Rayleigh fading is given by [34]

$$
\begin{aligned}
\bar{C}_{n_{\mathrm{T}_{0}}^{(\ell)}, n_{\mathrm{T}_{I}}^{(\ell)}, n_{\mathrm{R}}^{(\ell)}, \mathbf{P}^{(\ell)}}^{(\ell)}= & \mathcal{I}\left(n_{\mathrm{T}_{0}}^{(\ell)}+n_{\mathrm{T}_{I}}^{(\ell)}, n_{\mathrm{R}}^{(\ell)}, \tilde{\boldsymbol{\Psi}}^{(\ell)}\right) \\
& -\mathcal{I}\left(n_{\mathrm{T}_{I}}^{(\ell)}, n_{\mathrm{R}}^{(\ell)}, \boldsymbol{\Psi}^{(\ell)}\right),
\end{aligned}
$$

where $\mathbf{P}^{(\ell)}=\left[P_{0}^{(\ell)}, P_{1}^{(\ell)}, \ldots, P_{I^{(\ell)}}^{(\ell)}\right]^{T}$, and $P_{0}^{(\ell)}, P_{1}^{(\ell)}, \ldots, P_{I^{(\ell)}}^{(\ell)}$ are the averaged (over fading) power levels, received by the VAA, transmitted by the desired user $\left(P_{0}^{(\ell)}\right)$, and by the 
$I^{(\ell)}$ interferers. $\mathcal{I}\left(n_{\mathrm{T}}, n_{\mathrm{R}}, \boldsymbol{\Theta}\right)$ is the ergodic capacity of a single link MIMO system with $n_{\mathrm{T}}$ transmit and $n_{\mathrm{R}}$ receive antennas, when all the available radio resources are allocated to such link. The elements of the diagonal matrix $\Theta$ are the averaged power levels received by the VAA. More specifically [34],

$$
\boldsymbol{\Psi}^{(\ell)}=\frac{P_{1}^{(\ell)}}{\sigma_{\mathrm{N}}^{2}} \mathbf{I}_{n_{\mathrm{T}}} \oplus \frac{P_{2}^{(\ell)}}{\sigma_{\mathrm{N}}^{2}} \mathbf{I}_{n_{\mathrm{T}_{2}}} \oplus \cdots \oplus \frac{P_{I}^{(\ell)}}{\sigma_{\mathrm{N}}^{2}} \mathbf{I}_{n_{\mathrm{T}_{\mathrm{I}}}}
$$

and

$$
\tilde{\boldsymbol{\Psi}}^{(\ell)}=\frac{P_{0}^{(\ell)}}{\sigma_{\mathrm{N}}^{2}} \mathbf{I}_{n_{\mathrm{T}_{0}}} \oplus \boldsymbol{\Psi}^{(\ell)}
$$

where $\sigma_{\mathrm{N}}^{2}$ is the thermal noise power. Note that the eigenvalues of the matrices $\boldsymbol{\Psi}^{(\ell)}$ and $\tilde{\boldsymbol{\Psi}}^{(\ell)}$ have multiplicities larger than one.

We refer to [6] for the derivation of $\mathcal{I}\left(n_{\mathrm{T}}, n_{\mathrm{R}}, \boldsymbol{\Theta}\right)$ for the sake of brevity.

\subsection{Ergodic capacity between source and destination}

In the presence of a $Z$-hop communication protocol, the ergodic capacity from source to destination, denoted by $\bar{C}_{\mathrm{S}, \mathrm{D}}$, can be evaluated as the minimum of the values of the ergodic capacity in each link. By considering that each VAA uses one out of the $R$ available resources [4], the source-destination ergodic capacity can be written as

$$
\bar{C}_{\mathrm{S}, \mathrm{D}}=\min \left\{\frac{1}{R} \bar{C}_{n_{\mathrm{T}_{0}}^{(\ell)}, n_{\mathrm{T}_{I}}^{(\ell)}, n_{\mathrm{R}}^{(\ell)}, \mathbf{P}^{(\ell)}}^{(\ell)}\right\}_{\ell=1, . ., Z}
$$

where $\bar{C}_{n_{\mathrm{T}_{0}}, n_{\mathrm{T}_{I}}^{(\ell)}, n_{\mathrm{R}}^{(\ell)}, \mathbf{P}^{(\ell)}}^{(\ell)}$ is given by (3).

From (6) it can be noted that the parameter $R$ impacts on $\bar{C}_{\mathrm{S}, \mathrm{D}}$ as follows: on one hand the term $1 / R$ increases by getting $R$ smaller, on the other hand a decreasing of $R$ causes more interference (on average), therefore the capacity of the generic link gets smaller. This leads to the existence of an optimum value of $R$, as shown in the numerical results.

We assume that the contribution of an interfering VAA is significant only if its distance from the receiver VAA is lower than $p \cdot d$, where $p$ is a suitable integer, which depends on the propagation environment. Therefore, when $p=1$, only one-hop distant interferers are accounted for, whereas in the case $p=2$, one-hop and two-hop distant interferers are considered by the model. The latter assumption implies that there exists a maximum number of interfering VAAs on link $\ell$, denoted as $I_{\max }^{(\ell)}$.

\subsection{Outage probability definition}

Since the number of cooperating nodes is a random variable (rv), there is a nonzero probability that the source-destination mean capacity, $\bar{C}_{\mathrm{S}, \mathrm{D}}$, is lower than a given value, $C_{0}$, where the threshold $C_{0}$ depends on the specific application considered. Under such condition, we can introduce the following outage probability definition:

$$
P_{\text {out }} \triangleq \mathbb{P}\left\{\bar{C}_{\mathrm{S}, \mathrm{D}}<C_{0}\right\}
$$

\section{Performance analysis}

We now derive the metrics used to assess the performance of our VAA-based Framed ALOHA system: (a) the outage probability, as defined in (7); (b) the average throughput, and (c) the energy efficiency.

\subsection{Outage probability}

By applying the total probability theorem and by defining $\mathbf{E}=\left[n_{\mathrm{S}}, n_{\mathrm{R}_{1}}, \ldots, n_{\mathrm{R}_{Z-1}}, n_{\mathrm{D}}, I^{(1)}, \ldots, I^{(Z)}\right]^{T}$ and $\mathbf{e}=$ $\left[s, r_{1}, \ldots, r_{Z-1}, d, i^{(1)}, \ldots, i^{(Z)}\right]^{T}$, the outage probability can be evaluated as

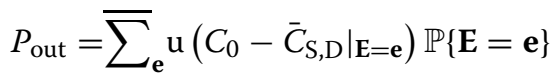

$$
\begin{aligned}
& =\overline{\sum_{\mathbf{e}}} \mathrm{u}\left(C_{0}-\left.\bar{C}_{\mathrm{S}, \mathrm{D}}\right|_{\mathbf{E}=\mathbf{e}}\right)\left(\prod_{\ell=1}^{Z} \mathbb{P}\left\{I^{(\ell)}=i^{(\ell)}\right\}\right) \\
& \times \mathbb{P}\left\{n_{\mathrm{S}}=s\right\} \mathbb{P}\left\{n_{\mathrm{D}}=d\right\} \prod_{k=1}^{Z-1} \mathbb{P}\left\{n_{\mathrm{R}, k}=r_{k}\right\},
\end{aligned}
$$

where

$$
\overline{\sum_{\mathbf{e}}} \triangleq \sum_{s=1}^{M+1} \sum_{r_{1}=1}^{M+1} \ldots \sum_{r_{Z-1}}^{M+1} \sum_{d=1}^{M+1} \sum_{i^{(1)}=0}^{I_{\max }^{(1)}} \sum_{i^{(2)}=0}^{I_{\max }^{(2)}} \ldots \sum_{i^{(Z)}=0}^{I_{\max }^{(Z)}},
$$

where $\bar{C}_{\mathrm{S}, \mathrm{D}}$ is given by (6) and $I_{\max }^{(\ell)}$ is the maximum number of interfering VAAs at the $\ell$ th hop.

It is easy to show that the number of interfering VAAs has a binomial distribution, whose probability function is

$$
\mathbb{P}\left\{I^{(\ell)}=i^{(\ell)}\right\}=\left(\begin{array}{c}
I_{\max }^{(\ell)} \\
i^{(\ell)}
\end{array}\right)\left(\frac{1}{R}\right)^{i^{(\ell)}}\left(1-\frac{1}{R}\right)^{I_{\max }^{(\ell)}-i^{(\ell)}}
$$

Note that $I_{\max }^{(\ell)}$ depends on $\ell$ and $p$ and can be written as

$$
I_{\max }^{(\ell)}= \begin{cases}p+\ell-1 & \text { for } \ell<p \\ 2 p-1 & \text { for } p \leq \ell \leq Z-(p+1) \\ p+Z-\ell-2 & \text { for } Z-(p+1)<\ell<Z-1 \\ p-1 & \text { for } \ell \geq Z-1\end{cases}
$$

Since the number of cooperating nodes is limited to a maximum value $M$, the distribution of the rvs $n_{\mathrm{S}}, n_{\mathrm{D}}$, and $n_{\mathrm{R}, k}(k=1, \ldots, Z-1)$ is no longer Poisson, but can be written as

$$
Q(i, N)= \begin{cases}P(i-1, N) & \text { for } i \leq M \\ 1-\sum_{l=0}^{M-1} P(l, N) & \text { for } i>M\end{cases}
$$


where $P(i, N) \triangleq \frac{N^{i}}{i !} e^{-N}$ is the Poisson distribution with parameter $N$.

Although exact, the evaluation of (8) becomes cumbersome as the number of hops increases. This can be easily understood by observing that the number of sums in (8) is equal to $Z(Z+1)$ and the complexity of the computation also depends on $M$ and $p$. Therefore, we propose two simpler approximated expressions for the outage probability. First, we can assume that the VAA size is independent at each hop. ${ }^{\mathrm{b}}$ Under this assumption, an approximate expression for the outage probability can be written as

$$
P_{\text {out }, \mathrm{A}}^{(\mathrm{app})}=1-\prod_{\ell=1}^{Z}\left(1-P_{\text {out }}^{(\ell)}\right),
$$

where $P_{\text {out }}^{(\ell)}$ is the probability that the capacity of the $\ell$ th link is lower than the threshold $C_{0}$, which varies with $\ell$, due to its dependance on the term $I_{\max }^{(\ell)}$. We also assume that all nodes transmit with the same power level, $P_{\mathrm{T}}$, so that the averaged (over fading) power received by a given node located at distance $x$ is $P_{\mathrm{T}} x^{-\beta} / k$. Using the total probability theorem and defining $m \triangleq$ $I_{\max }^{(\ell)}, \boldsymbol{\Omega}^{(\ell)}=\left[I^{(\ell)}, n_{\mathrm{T}_{0}}^{(\ell)}, n_{\mathrm{T}_{1}}^{(\ell)}, \ldots, n_{\mathrm{T}_{\mathrm{m}}}^{(\ell)}, n_{\mathrm{R}}^{(\ell)}\right]^{T}$ and $\omega=$ $\left[i, a_{0}, a_{1}, \ldots, a_{m}, r\right]^{T}$, the outage probability at the $\ell$ th hop can be written as

$$
\begin{aligned}
P_{\text {out }}^{(\ell)}= & \bar{\sum}_{\omega} \mathrm{u}\left(C_{0}-\frac{1}{R} \bar{C}_{a_{0}, a_{I}, r, \mathbf{P}^{(\ell)} \mid \boldsymbol{\Omega}^{(\ell)}=\omega}^{(\ell)}\right) \mathbb{P}\left\{\boldsymbol{\Omega}^{(\ell)}=\omega\right\} \\
= & \bar{\sum}_{\omega} \mathrm{u}\left(C_{0}-\frac{1}{R} \bar{C}_{a_{0}, a_{I}, r, \mathbf{P}^{(\ell)} \mid \boldsymbol{\Omega}^{(\ell)}=\omega}^{(\ell)}\right) \mathbb{P}\left\{I^{(\ell)}=i\right\} \\
& \times Q\left(a_{0}, N\right)\left(\prod_{i=1}^{\mathrm{m}} Q\left(a_{i}, N\right)\right) Q(r, N),
\end{aligned}
$$

where $a_{I} \triangleq \sum_{j=1}^{I^{(\ell)}} a_{j}, Q(i, N)$ is given by (11), and

$$
\overline{\sum_{\omega}} \triangleq \sum_{i=0}^{\mathrm{m}} \sum_{a_{0}=1}^{M+1} \sum_{a_{1}=1}^{M+1} \ldots \sum_{a_{\mathrm{m}}=1}^{M+1} \sum_{r=1}^{M+1}
$$

We define $\boldsymbol{a}=\left[a_{1}, a_{2}, . ., a_{m}\right]^{T}, \mathbf{P}_{I}^{(\ell)}=\left[P_{1}^{(\ell)}, P_{2}^{(\ell)}, . ., P_{m}^{(\ell)}\right]^{T}$, and $\boldsymbol{F}_{(t)}$, with an $m \times m$ matrix having $t$ elements per column equal to ' 1 ' and the remaining elements equal to ' 0 ', such that the different columns contain all the possible, non repeated, combinations of the positions of the '1. We also denote as $\boldsymbol{f}_{j,(t)}$ the $j$ th column of $\boldsymbol{F}_{(t)}$, that is, $\boldsymbol{F}_{(t)}=\left[\boldsymbol{f}_{1,(t)} \boldsymbol{f}_{2,(t)} \cdot \boldsymbol{f}_{m,(t)}\right]$.

As an example, in the case of $m=3$, we have that $\boldsymbol{F}_{(1)}=$ $\left[\begin{array}{lll}1 & 0 & 0 \\ 0 & 1 & 0 \\ 0 & 0 & 1\end{array}\right]$ and $\boldsymbol{F}_{(2)}=\left[\begin{array}{lll}1 & 0 & 1 \\ 1 & 1 & 0 \\ 0 & 1 & 1\end{array}\right]$. Expression (13) can be simplified using the following identity

$$
\begin{aligned}
\mathrm{u}\left(C_{0}-\right. & \left.\frac{1}{R} \bar{C}_{a_{0}, a_{I}, r, \mathbf{P}^{(\ell)} \mid \boldsymbol{\Omega}^{(\ell)}=\omega}^{(\ell)}\right) \mathbb{P}\left\{I^{(\ell)}=i\right\} \\
= & \left(\frac{1}{R}\right)^{i}\left(1-\frac{1}{R}\right)^{\mathrm{m}-i} \\
& \sum_{j=1}^{\left(\begin{array}{c}
\mathrm{m} \\
i
\end{array}\right)} \mathrm{u}\left(C_{0}-\frac{1}{R} \bar{C}_{a_{0}, \boldsymbol{a}^{T} \cdot \boldsymbol{f}_{j,(i)}^{(\ell)}, r,\left[P_{0}^{(\ell)}, \mathbf{P}_{\mathrm{I}}^{(\ell) T} \cdot f_{j,(i)}\right]}\right)
\end{aligned}
$$

In the case of $i=0$ (i.e., no interferers), the sum in (15) is equal to $\mathrm{u}\left(C_{0}-\frac{1}{R} \bar{C}_{a_{0}, r,\left[P_{0}^{(\ell)}\right]}^{(\ell)}\right)$, with $\tilde{\boldsymbol{\Psi}}=$ $\frac{P_{0}}{\sigma_{\mathrm{N}}^{2}} \mathbf{I}_{n_{\mathrm{T}_{0}}}$. In the case of $i=m$, the sum (15) is equal to $\mathrm{u}\left(C_{0}-\frac{1}{R} \bar{C}_{a_{0}, \sum_{i=1}^{(\ell)} a_{i}, r,\left[P_{0}^{(\ell)}, . . P_{\mathrm{m}}^{(\ell)}\right]}^{\mathrm{m}}\right)$.

Approximation (12) can be further simplified by neglecting the fact that some VAA links close to the source and destination will be affected by a reduced number of interferers compared to VAA links located in the center of the transmission chain (i.e., we neglect border effects). This can be easily understood by looking at (10), where the maximum number of interfering VAAs is evaluated as a function of $\ell$. Under such assumption, $P_{\text {out }}^{(\ell)}=\hat{P}_{\text {out }} \forall \ell$, and the upper bound for the overall outage probability can be written as

$$
P_{\text {out }, \mathrm{B}}^{\text {(app) }}=1-\left(1-\hat{P}_{\text {out }}\right)^{Z},
$$

where $\hat{P}_{\text {out }}$ represents the outage probability of each link. $\hat{P}_{\text {out }}$ can be evaluated using (13) with $m=2 p-1$. The impact of approximations A and B will be evaluated through the numerical results.

\subsection{Average throughput}

Another metric considered in this paper is the average throughput, $T$, defined as the source-destination throughput averaged over nodes' location and normalized with respect to the channel bandwidth. This metric can be evaluated as

$$
T=\sum_{i=1}^{n_{\max }-1} f\left(c_{i}\right) c_{i}+\left(1-\sum_{i=1}^{n_{\max }-1} f\left(c_{i}\right)\right) c_{n_{\max }}[\mathrm{bit} / \mathrm{s} / \mathrm{Hz}],
$$

where $f\left(c_{i}\right) \triangleq \mathbb{P}\left\{\bar{C}_{\mathrm{S}, \mathrm{D}}=c_{i}\right\}=F\left(c_{i}\right)-F\left(c_{i-1}\right)$ is the probability function of $\bar{C}_{\mathrm{S}, \mathrm{D}}$, and $F\left(c_{i}\right)$ is the complementary cumulative function of $\bar{C}_{\mathrm{S}, \mathrm{D}}$ given by

$$
F\left(c_{i}\right)=\mathbb{P}\left\{\bar{C}_{\mathrm{S}, \mathrm{D}} \geq c_{i}\right\}=P_{\text {in }}=1-P_{\text {out }}
$$

where, obviously, for the outage probability we can use the exact formula, $P_{\text {out }}$ (given by (8)), or one of its approximations, $P_{\text {out }, \mathrm{A}}^{(\mathrm{app})}$ or $P_{\mathrm{out}, \mathrm{B}}^{(\mathrm{app})}$. Finally, $c_{n_{\max }}$ is the maximum value the average capacity may assume. 


\subsection{Energy efficiency}

We evaluate the energy efficiency, defined as

$$
\xi \triangleq \frac{T}{\bar{E}}=\frac{T}{T_{p} 2(N+1) P_{T} Z}[\mathrm{bit} / \mathrm{s} / \mathrm{Hz} / \mathrm{J}],
$$

where $\bar{E}$ is the (average) energy required to transmit a packet from source to destination; $T_{p}$ is the transmission time of the packet, $N$ is the mean number of cooperating nodes in a VAA (therefore, $N+1$ is the average size of a VAA).

$\xi$ is related to the energy efficiency of the multi-hop $\mathrm{V}$-MIMO system and represents the ratio between the average throughput obtainable and the energy spent for the transmission of a packet from source to destination through the $Z$ hops. To evaluate $\bar{E}$, we have assumed that the same amount of energy is necessary to transmit or receive a packet (this justifies the term 2 at the denominator of (19)); this assumption appears to be reasonable for many hardware platforms. ${ }^{\mathrm{c}}$

\section{Numerical results}

Results are obtained by considering, if not otherwise specified, $\rho=2 \cdot 10^{-2} \mathrm{~m}^{-2}, \sigma_{\mathrm{N}}^{2}=8 \cdot 10^{-15} \mathrm{~W}$, and $M=4$. We consider two different channel models for intra-VAA and inter-VAAs communication. In the former case, we set $k_{0}=41 \mathrm{~dB}, k_{1}=13.03(\beta=3)$, and $L_{\text {th }}=70 \mathrm{~dB}$; while in the case of the inter-VAA communication channel, we set $k_{0}=15 \mathrm{~dB}$ and we vary $\beta\left(k_{1}\right)$ for the inter-VAAs transmission $\left(L_{\text {th }}\right.$ is not fixed in this case, since we assume that the different VAAs are always connected). The signalto-noise ratio (SNR) $\gamma$ is defined as $P_{0} / \sigma_{\mathrm{N}}^{2}$, being $P_{0}$ the averaged (over fading) useful receive power, which is given by $P_{\mathrm{T}} d^{-\beta} / k$.

\subsection{Performance of the $\ell$ th hop}

In this section we evaluate the performance of the generic $\ell$ th hop, in terms of the complementary outage probability, $P_{\text {in }}^{(\ell)}=1-P_{\text {out }}^{(\ell)}$, where $P_{\text {out }}^{(\ell)}$ is given by (13), when considering the worst case that is a central link affected by the maximum number of interferers.

Figure 2 shows $P_{\text {in }}^{(\ell)}$ as a function of the requirement on the capacity, for different values of $R$, having set $p=2$, $\beta=3$ and $\gamma=10 \mathrm{~dB}$. Note that the number of transmit and receive antennas of the various hops is a discrete rv; as a consequence, the source-destination capacity is a discrete rv, too. This justifies the step-wise behavior of the curves. All curves show a similar behavior, with $P_{\text {in }}^{(\ell)}$ monotonically decreasing. $P_{\text {in }}^{(\ell)}$ increases for smaller $R$ because the SNR is low (10 dB), and the system tends to be noise-limited. As a consequence, interference does not play a major role on the system performance and

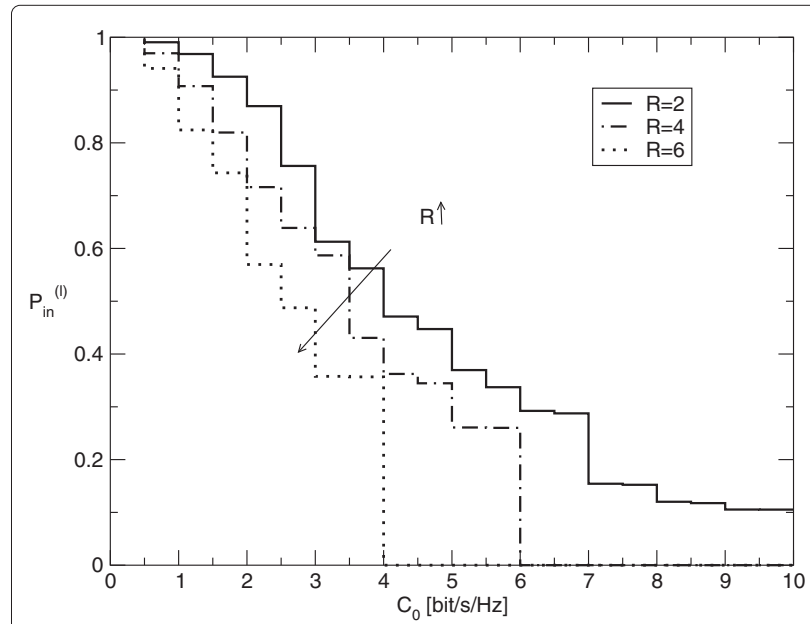

Figure $2 P_{\text {in }}^{(\ell)}$ as a function of $C_{0}$, when $\gamma=10 \mathrm{~dB}$ and $\beta=3$.

the behavior of the source-destination capacity mainly depends on the term $1 / R$ (see (6)). Small values of $R$ increase the amount of interference, but this is still negligible compared to thermal noise. On the contrary, the corresponding performance increases since the amount of radio resources available to each VAA link increases as $R$ decreases. When the SNR is large, interference plays a more significant role with respect to noise. The latter behavior is shown in Figure 3, where $\gamma=40 \mathrm{~dB}$ is considered. When the capacity threshold is low (approximatively lower than $2 \mathrm{bit} / \mathrm{s} / \mathrm{Hz}$ ), the term $1 / R$ still dominates the behavior of $\bar{C}_{\mathrm{S}, \mathrm{D}}$, while for larger value of $C_{0}$, an increase of $R$ allows to decrease the level of interference at each link, resulting in better performance.

Figure 4 compares the performance obtained with framed ALOHA to the case of a perfect centralized (deterministic) resource assignment, having set $\gamma=10$ $\mathrm{dB}, \beta=3$, and $p=2$ in the case of FA. In the case

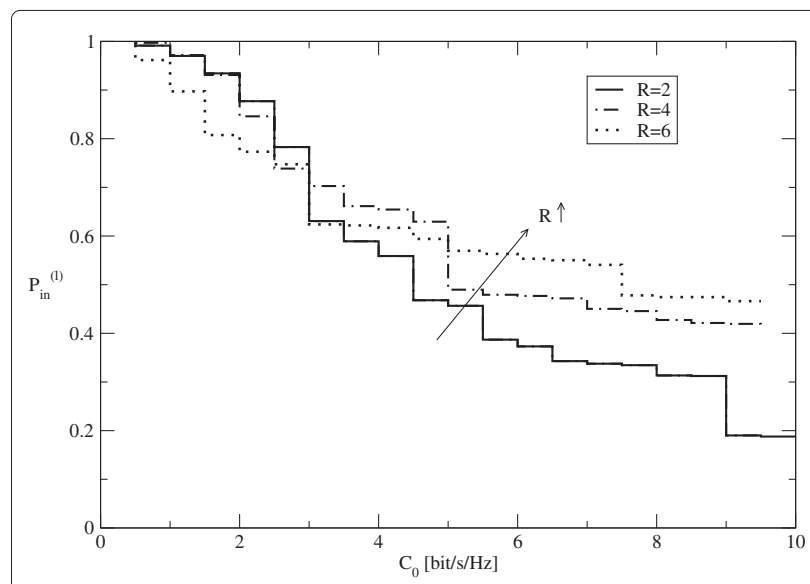

Figure $3 P_{\text {in }}^{(\ell)}$ as a function of $C_{0}$, when $\gamma=40 \mathrm{~dB}$ and $\beta=3$. 


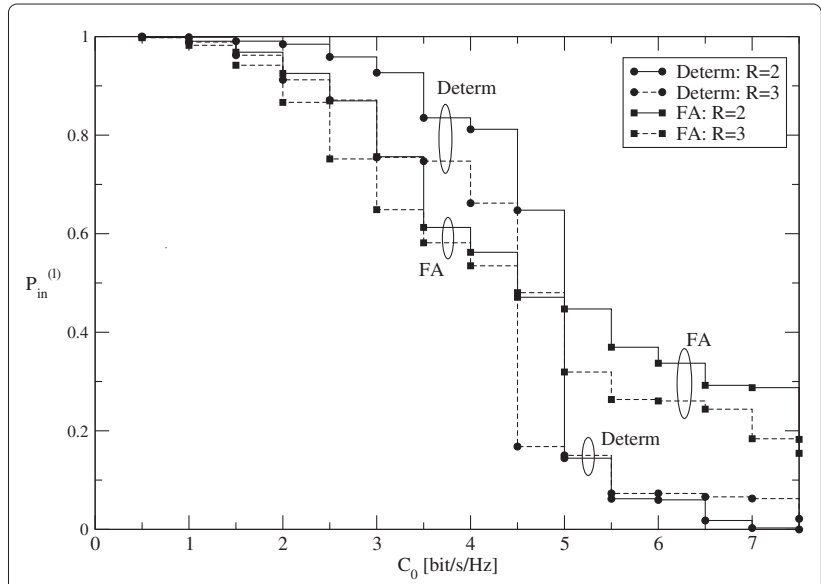

Figure $4 P_{\text {in }}^{(\ell)}$ as a function of $C_{0}$, when $\gamma=10 \mathrm{~dB}$ and $\beta=3$. of deterministic resource allocation, we assume that the resources are assigned to the VAAs in order to maximize the distance between two interfering VAAs. More specifically, when $R=2$, the links $(\ell-2)$, $(\ell),(\ell+2)$ use the first resource and $(\ell-3),(\ell-1),(\ell+1)$ use the second one. As expected, for the values of outage probability of interest (i.e., $P_{\text {in }}^{(\ell)} \approx 0.9$ ), the centralized approach outperforms framed ALOHA. On the other hand, if the capacity requirement is very high, framed ALOHA can provide better performance, thanks to the randomness of the interfering VAAs. On the contrary, in the deterministic case when for example, in $R=2$, the one-hop distant VAA will interfere with probability equal to one.

Figure 5 shows $P_{\text {in }}^{(\ell)}$ against $R$ for different values of the capacity requirement and $\gamma$, in the case of framed ALOHA with $p=2$, having set $\beta=3$. When $C_{0}$ is small, the optimum value of $R$ is 2 ; on the other hand, when $C_{0}=$ $10 \mathrm{bit} / \mathrm{s} / \mathrm{Hz}$, its performance is optimized by choosing $R=5$. This optimum value is a trade-off between the need

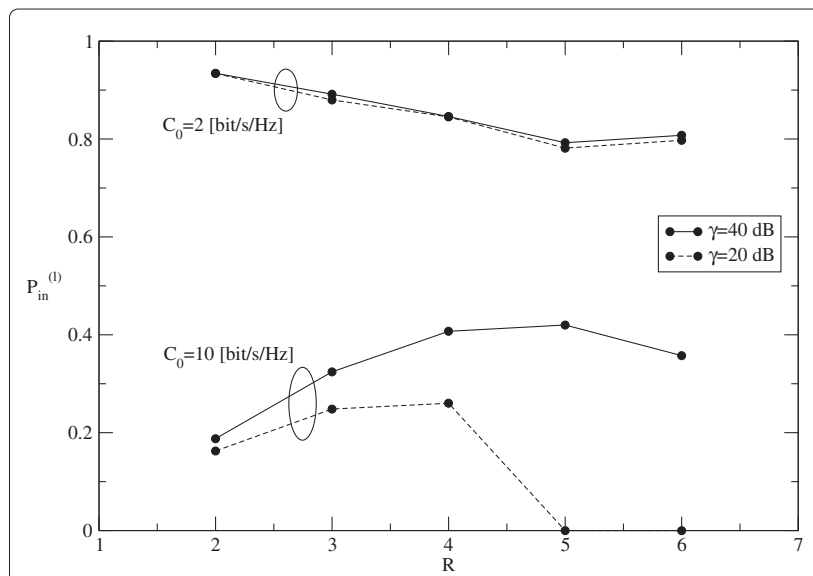

Figure $5 P_{\text {in }}^{(\ell)}$ as a function of $R$ when $\beta=3$, for framed ALOHA.

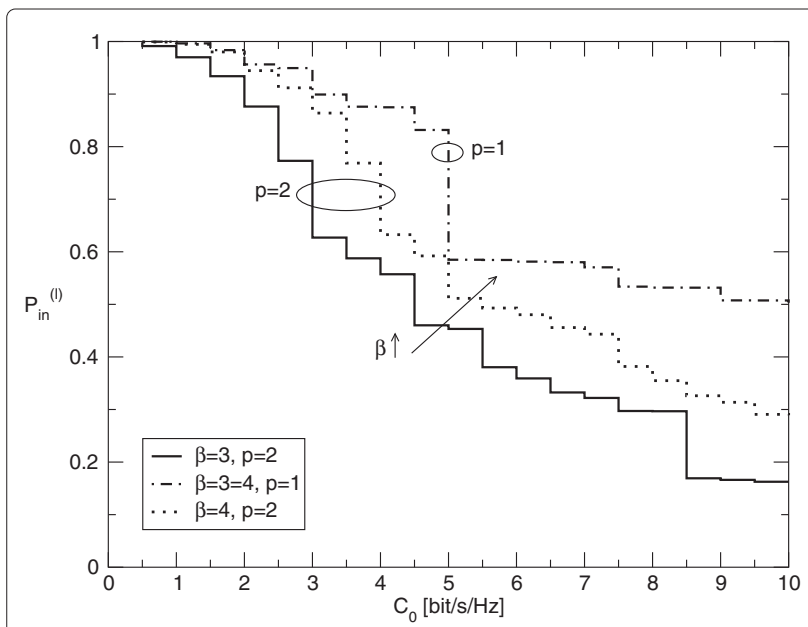

Figure $6 P_{\text {in }}^{(\ell)}$ as a function of $C_{0}$, when $R=2$ and $\gamma=20 \mathrm{~dB}$.

of reducing the impact of interference and that of increasing the amount of radio resources to be used. As expected, the optimum $R$ tends to become smaller by decreasing the SNR.

The impact of the value of $p$ on the system performance is considered in Figure 6, where the complementary outage probability for the generic link, $P_{\text {in }}^{(\ell)}$, is shown as a function of $C_{0}$, when varying $p$ and $\beta$. Other parameters are $\mathrm{SNR}=20 \mathrm{~dB}$ and $R=2$. By increasing $\beta$, the corresponding signal to interference ratio increases, as a consequence, the curve related to $p=2$ approaches the ones related to $p=1$. Note also that when $p=1, P_{\text {in }}^{(l)}$ does not depend on $\beta$, being the interferer and as useful VAAs at the same distance from the receiver VAA (i.e., the signal to interference ratio does not depend on $\beta$ ). Finally, the curves are approximatively coincident when $P_{\text {in }}^{(\ell)}>0.9$, which represents the zone of interest.

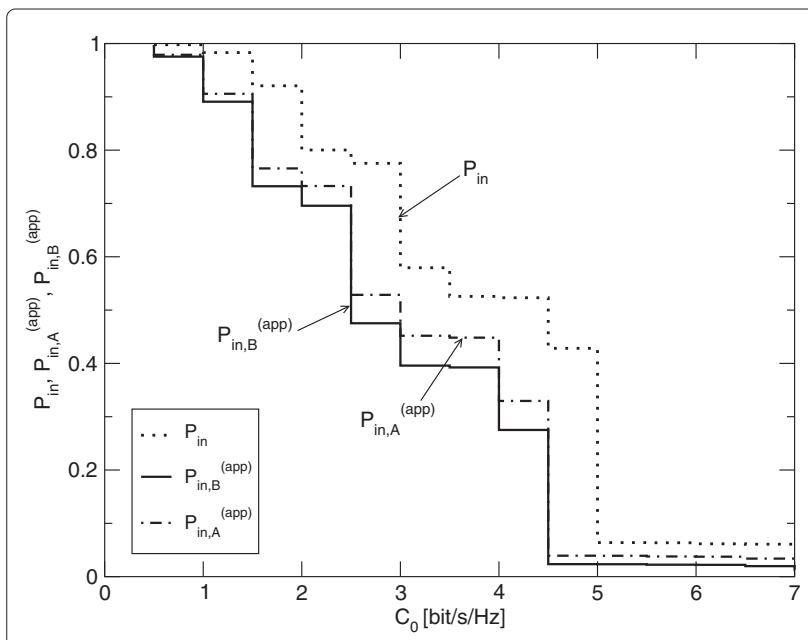

Figure $7 P_{\text {in }}$ and its approximations as a function of $C_{0}$. 


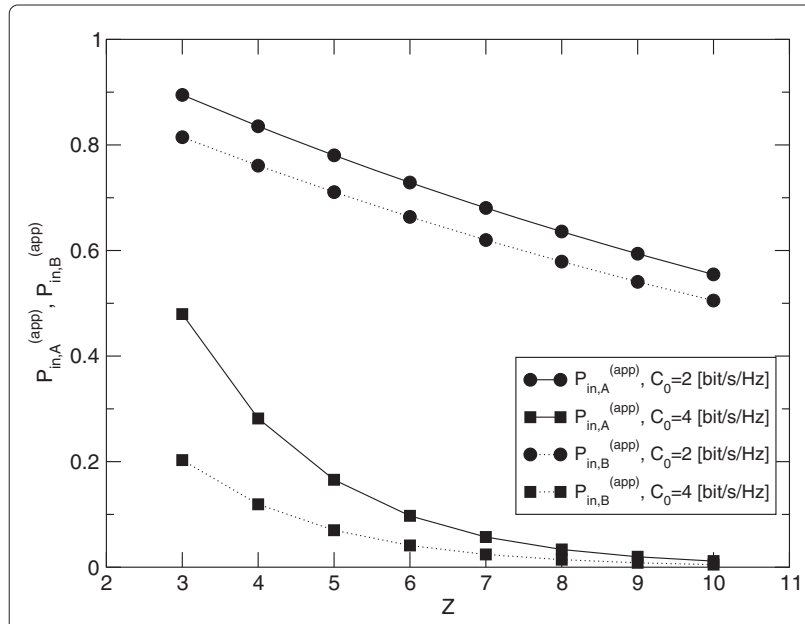

Figure $8 P_{\mathrm{in}, \mathrm{A}}^{(\mathrm{app})}$ and $P_{\mathrm{in}, \mathrm{B}}^{(\mathrm{app})}$ as a function of $\mathrm{Z}$, when $\gamma=20 \mathrm{~dB}$ and $\beta=3$.

\subsection{Performance of the whole transmission chain}

This section considers the whole transmission chain by investigating the behavior of the following metrics: (a) the exact overall complementary outage probability, $P_{\text {in }}=$ $1-P_{\text {out }}$ (b) the approximated overall complementary out-

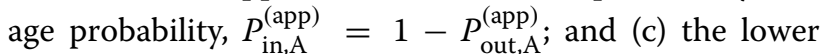
bound for the overall complementary outage probability, $P_{\text {in, } \mathrm{B}}^{(\mathrm{app})}=1-P_{\text {out, } \mathrm{B}}^{(\mathrm{app})}$.

The tightness of the approximations $\mathrm{A}$ and $\mathrm{B}$ is shown in Figure 7, where we compare the exact formula for $P_{\text {in }}$, obtained with (13), with the approximations $P_{\text {in, }, A}^{(\text {app }}$ and $P_{\text {in }, B}^{\text {(app) }}$ (evaluated using (12) and (16), respectively). Due to the computation complexity in evaluating $P_{\text {in }}$ given by (13), we set $p=1$ and $Z=7$; moreover, $R=2$ and

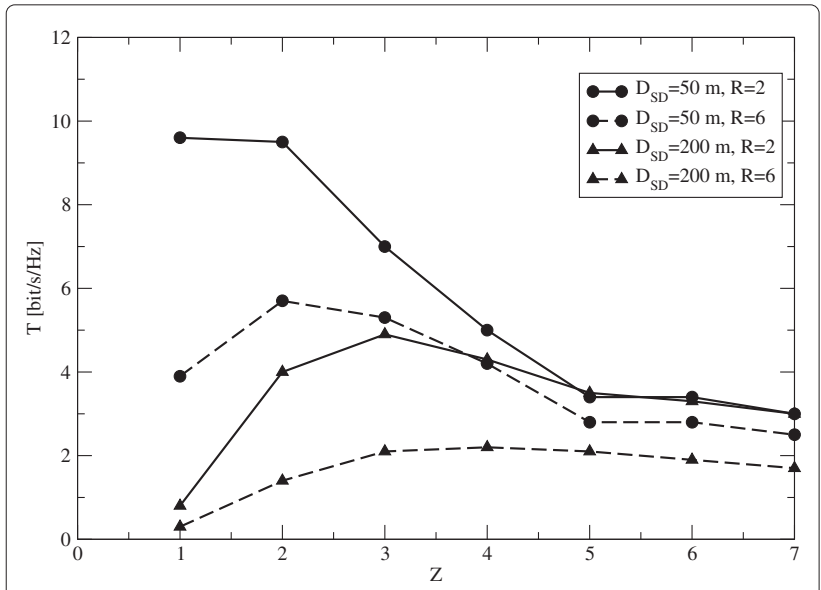

Figure $9 T$ as a function of $Z$, when $p=2$, for different value of $D_{\mathrm{SD}}$ and $R$.

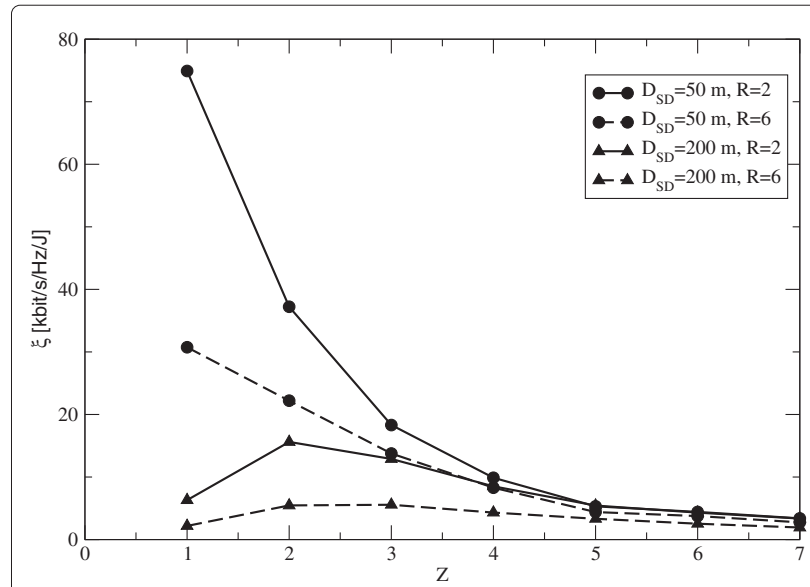

Figure $10 \xi$ as a function of $Z$, when $p=2$, for different value of $D_{\text {SD }}$ and $R$.

$\beta=3$ are set. When the links are assumed to be independent (assumption is used to obtain approximations A and B), a lower complementary outage probability is obtained. This can be justified by observing that in such condition, there is a larger probability to find at least one link in outage. As expected, the curve obtained with approximation $\mathrm{A}$ is tighter than approximation $\mathrm{B}$ (which neglects border affects).

In Figure 8 we show the overall complementary outage probability, given by the approximated formulas, $P_{\text {in, A }}^{(\text {app }}$ and $P_{\mathrm{in}, \mathrm{B}}^{(\mathrm{app})}$, against $Z$ for different values of $C_{0}$, having set $p=2, \gamma=20 \mathrm{~dB}, R=2$, and $\beta=3$. We can note that the complementary outage probability decreases when $Z$ gets larger and that the two approximations get closer when $Z$ increases and/or $C_{0}$ decreases.

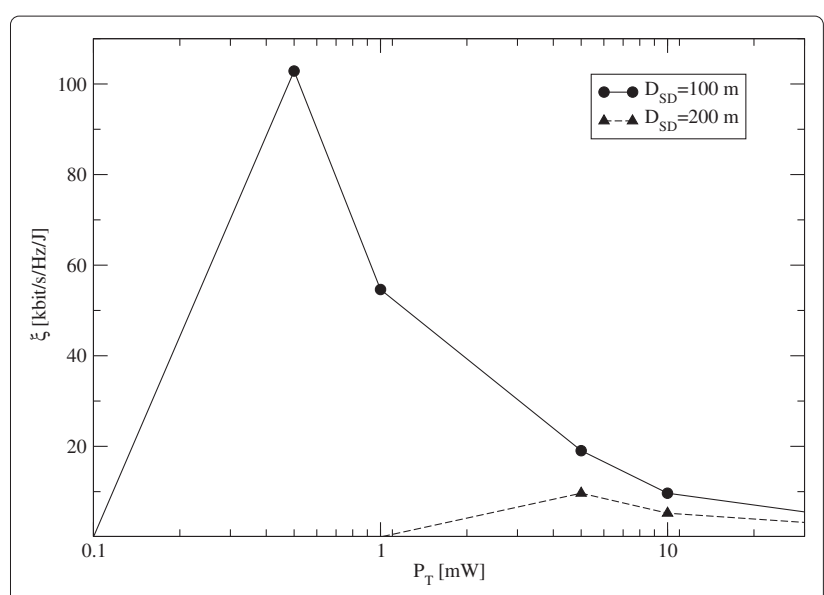

Figure $11 \xi$ as a function of $P_{\mathrm{T}}$, when $p=2$ and $Z=4$, for different value of $D_{\text {SD }}$. 
In the following figures, we set $\beta=4, p=2$, and $c_{n_{\max }}=$ $10 \mathrm{bit} / \mathrm{s} / \mathrm{Hz}$, and we use the approximated formula $P_{\text {in, A }}^{\text {(app) }}$ to compute $T$.

In Figure 9 and Figure 10 we show the average throughput, $T$, and the energy efficiency, $\xi$, as a function of the number of hops, $Z$, for different values of $D_{\mathrm{SD}}$ and $R$, respectively. In the figures we set $P_{\mathrm{T}}=10 \mathrm{dBm}$ and, once $D_{\mathrm{SD}}$ is fixed, the SNR at the VAAs will change by varying $Z$ : by increasing $Z$, the VAAs distance, $d$, decreases, resulting in a larger signal-to-noise ratio. Figure 9 shows that the average throughput presents a maximum, which can be explained as follows. For small values of $Z$, the system is noise-limited, whereas for larger $Z$ the SNR increases and the system becomes interference-limited; hence, an increase of $Z$ causes a decrease of the signal-tointerference ratio. In the case $D_{\mathrm{SD}}=50 \mathrm{~m}$ and $R=2$, the maximum is not present because the system is mainly interference-limited (few slots are available in the frame, while the SNR is large also for small value of $Z$ ). Almost the same holds for the behavior of $\xi$. Compared to the average throughput case, here the maxima are reached for lower values of $Z$. This is due to the additional amount of energy spent when $Z$ increases. This also explains the fact that there is no maximum when $D_{\mathrm{SD}}=50 \mathrm{~m}$.

Finally, $\xi$ as a function of $P_{\mathrm{T}}$, for $Z=4$ and $T_{p}=1$ ms and varying $D_{\mathrm{SD}}$, is illustrated in Figure 11 . The curves present a maximum, meaning that once $D_{\mathrm{SD}}$ and nodes density are fixed, there exists an optimum transmit power maximizing $\xi$. Such optimum is obtained when the tradeoff between energy consumption and SNR is reached.

\section{Conclusions}

A framed ALOHA multi-hop V-MIMO system is investigated. Random location of cooperating nodes as well as propagation environment are considered and their effects on the overall performance are evaluated. Several performance metrics are considered to assess the sourcedestination performance: outage probability, which is the probability that the capacity between source and destination is smaller than a given threshold, and energy efficiency. The proposed framework allows us to optimize different system parameters, such as the number of relays to be distributed between source and destination nodes, the number of radio resources, $R$, and the transmit power. Finally, we would underline that the presented framework could be easily extended to the case of other possible contention-based protocols, as carrier sense multiple access-based protocols by properly changing (9) based on the protocol considered.

\section{Endnotes}

a As discussed in [6], the use of other models is straightforward. b Under such assumption, we neglect the correlation existing between the size of the receive VAA at the $\ell$ th link and the size of the transmit VAA at the $(\ell+1)^{\text {th }}$ link. More specifically, two uncorrelated sets of cooperative nodes are chosen by each VAA for receive (from the previous VAA) and transmit (to the subsequent VAA) purposes.

c Through this definition, we do not account for the energy which is not radiated. Though this component is normally relevant, it would only affect $\xi$ through a proper down-scaling. For the purpose of this paper, such downscaling of the absolute value of $\xi$ is not conceptually relevant.

\section{Competing interests}

The authors declare that they have no competing interests.

\section{Author details}

${ }^{1}$ RadioNetworks, DEl, University of Bologna, Bologna, Italy. ${ }^{2}$ National Research Council (CNR), IEIIT, Torino, Italy.

Received: 19 October 2012 Accepted: 4 April 2013

Published: 1 May 2013

\section{References}

1. GJ Foschini, Layered space-time architecture for wireless communication in a fading environment when using multi-element antennas. Bell Labs Tech. J. 1(2), 41-59 (1996)

2. E Telatar, Capacity of multi-antenna Gaussian channels. Eur. Trans. Telecommun. 10(6), 585-595 (1999)

3. M Dohler, A Gkelias, H Aghvami, Resource allocation for FDMA-based regenerative multi-hop links. IEEE Trans. Wireless Commun. 3, 1989-1993 (2004)

4. M Dohler, A Gkelias, H Aghvami, A resource allocation strategy for distributed MIMO multi-hop communication systems. IEEE Commun. Lett. 8, 99-101 (2004)

5. H Zhang, H Dai, in Proceedings of the Conference on Information Sciences and Systems, CISS 2004. On the capacity of distributed MIMO systems (Princeton University, March 2004), pp. 17-19

6. C Buratti, A Zanella, Multi-hop virtual MIMO systems with channel reuse in a Poisson field of nodes. IEEE Trans. Veh. Technol. 60(5), 2060-2069 (2011)

7. AA Dowhuszko, G Corral-Briones, J Hamalainen, R Wichman, On throughput-fairness tradeoff in virtual MIMO systems with limited feedback. EURASIP J. Wireless Comm. Netw. (ID 102064), 17 (2009)

8. V Jimenez, C Ribeiro, A Gameiro, G Armada, Effects of channel estimation on multiuser virtual MIMO-OFDMA relay-based networks. Eurasip J. Wireless Comm. Netw. (ID 764784), 13 (2009)

9. Y Yuan, Z He, M Chen, Virtual MIMO-based cross-layer design for wireless sensor networks. IEEE Trans. Veh. Technol. 55, 856-864 (2006)

10. X Li, Energy efficient wireless sensor networks with transmission diversity. IEEE Electron. Lett. 39, 1753-1755 (2003)

11. S Cui, AJ Goldsmith, A Bahai, Energy-efficiency of MIMO and cooperative MIMO techniques in sensor networks. IEEE J. Sel. Areas Commun. 22, 1089-1098 (2004)

12. SK Jayaweera, Virtual MIMO-based cooperative communication for energy-constrained wireless sensor networks. IEEE Trans. Wireless Commun. 5(5) (2006)

13. W Deng, $X$ Gao, in Proceedings of International Symposium on Intelligent Signal Processing and Communication Systems (ISPACS 2007). Three-hop cooperative diversity system and symbol error rate analysis ( Huaqiao University, Xiamen, 28 November to 1 December 2007)

14. Y Lang, D Wubben, C Bockelmann, KD Kammeyer, in Proceedings of the 6th International Symposium on Modeling and Optimization in Mobile, Ad Hoc, and Wireless Networks, WIOPT 2008. A closed power allocation solution for outage restricted distributed MIMO multi-hop networks (Berlin, 1), pp. 65-70 
15. MD Renzo, F Graziosi, F Santucci, in Proceedings of IEEE of ICC Beijing, 19-23 May 2008. On the performance of CSI-assisted cooperative communications over generalized fading channels, pp. 1001-1007

16. H Gharavi, B Hu, N Wu, in Proceedings of IEEE ICC 2010. A design framework for high-density wireless ad-hoc networks achieving cooperative diversity (Cape Town, =, May 2010), pp. 23-27

17. AD Coso, U Spagnolini, C Ibars, Cooperative distributed MIMO channels in wireless sensor networks. IEEE J. Sel. Areas Commun. 25, 402-414 (2007)

18. J Liang, Q Liang, Channel selection in virtual MIMO wireless sensor networks. IEEE Trans. Veh. Technol. 58, 2249-2257 (2009)

19. G Bravos, AG Kanatas, Energy efficiency comparison of MIMO-based and multihop sensor networks. Eurasip J. Wireless Comm. Netw. 732145 (2008)

20. MKTsatsanis, R Zhang, S Banerjee, Network-assisted diversity for random access wireless networks. IEEE Trans. Signal Process. 48(3), 702-711 (2000)

21. R Zhang, MK Tsatsanis, Network-assisted diversity for random access in dispersive channels. IEEE Trans. Commun. 50(4), 623-632 (2002)

22. R Lin, A Petropulu, A new wireless network medium access protocol based on cooperation. IEEE Trans. Signal Process. 53(12), 4675-4684 (2005)

23. H Guo, H Hu, Y Zhang, HH Chen, Adaptive space-time diversity Slotted ALOHA over $2 \times m$ MIMO multi-access channels. IEEE Trans. Veh. Technol. 58(7), 3271-3282 (2009)

24. Y Lin, V Wong, Cross-layer design of MIMO-enabled WLANs with network utility maximization. IEEE Trans. Veh. Technol. 58(5), 2443-2456 (2009)

25. D Qian, D Zheng, J Zhang, N Shroff, in Proceedings of IEEE INFOCOM 29th Annual Joint Conference. CSMA-Based distributed scheduling in multi-hop MIMO networks under SINR model (San Diego, CA, 1)

26. M Martalò, C Buratti, G Ferrari, R Verdone, Decentralized detection in IEEE 802.15.4 wireless sensor networks. EURASIP J. Wireless Commun. Netw. 2010(10) (2010)

27. M Martalò, C Buratti, G Ferrari, R Verdone, Clustered IEEE 802.15.4 sensor networks with data aggregation: energy consumption and probability of error. IEEE Wireless Commun. Lett. 2, 71-74 (2013)

28. C Buratti, A Zanella, R Verdone, in Proceedings of International Conference on Cognitive Radio Oriented Wireless Networks (CROWNCOM) 2010. On the capacity of slotted ALOHA-based multi-hop Virtual MIMO systems (Cannes, June 2010), pp. 9-11

29. C Buratti, A Zanella, R Verdone, in Proceedings of Future Network and Mobile Summit (FUNEMS) 2010. Throughput analysis of slotted ALOHA multiple hop systems with virtual antenna arrays ( Firenze, Italy, June 2010), pp. 16-18

30. RA Horn, CR Johnson, (Cambridge University Press, Cambridge, 1st edn., 1990)

31. TA Cover, JA Thomas, Elements of Information Theory, (Wiley, New York, 1st edn., 1991)

32. J Kingman, (Oxford University Press, Oxford,1993)

33. LG Roberts, ALOHA packet system with and without slots and capture. ACM SIGCOMM Comput. Commun. 5(2), 28-42 (1975)

34. M Chiani, MZ Win, H Shin, MIMO networks: the effects of interference. IEEE Trans. Inf. Theory. 56, 336-349 (2010)

doi:10.1186/1687-1499-2013-118

Cite this article as: Buratti et al:: Performance analysis of multi-hop framed ALOHA systems with virtual antenna arrays. EURASIP Journal on Wireless Communications and Networking 2013 2013:118.

\section{Submit your manuscript to a SpringerOpen ${ }^{\odot}$ journal and benefit from:}

- Convenient online submission

Rigorous peer review

- Immediate publication on acceptance

- Open access: articles freely available online

- High visibility within the field

- Retaining the copyright to your article

Submit your next manuscript at $\boldsymbol{\wedge}$ springeropen.com 